\title{
Reconhecimento e notificação de violência pelos profissionais da estratégia de saúde da família
}

\author{
Recognition and reporting of violence \\ by professionals of the family health strategy \\ Reconocimiento y reporte de violencia por parte de los profesionales \\ de la estrategia de salud de la familia \\ Cléa Adas Saliba GARBIN ${ }^{1}$ \\ Tânia Adas Saliba ROVIDA² \\ Adriana Alves COSTA ${ }^{3}$ \\ Artênio José Isper GARBIN ${ }^{4}$
${ }^{I}$ Doutora em Odontologia Legal e Deontologia. Professora Titular do Departamento de Odontologia Infantil e Social da Universidade Estadual Paulista. Araçatuba, SP, Brasil. cgarbin@foa.unesp.br Universidade Estadual Paulista. Araçatuba, SP, Brasil.tasalibarovida@foa.unesp.br ${ }^{4}$ Doutor em Ortodontia. Professor Adjunto do Departamento de Odontologia Infantil e Social da \\ ${ }^{2}$ Doutora em Odontologia Legal e Deontologia. Professora Assistente Doutora do Departamento de Odontologia Infantil e Social da \\ ${ }^{3}$ Mestranda do Curso de Odontologia da Universidade Estadual Paulista. Araçatuba, SP, Brasil.adryana_alves@hotmail.com \\ Universidade Estadual Paulista. Araçatuba, SP, Brasil.agarbin@foa.unesp.br
}

\begin{abstract}
Resumo
A violência tem se apresentado como uma demanda marcante aos setores sanitários. Objetivou-se verificar se os profissionais da Estratégia Saúde da Família (ESF) de um município de São Paulo encontram dificuldades quanto ao processo de reconhecimento e notificação das ocorrências envolvendo violência. Trata-se de um estudo transversal e descritivo. Do total de 135 profissionais, 57 consentiram e responderam um questionário autoaplicado com questões abertas e fechadas. Os dados foram analisados quantiqualitativamente. $75,4 \%$ dos profissionais não conhecem a ficha de notificação de violências; $60 \%$ não se sentem responsáveis pela notificação dos casos. O acontecimento da violência intrafamiliar, muitas vezes, está associado ao uso de drogas e/ou álcool e também da condição socioeconômica dos indivíduos envolvidos. Conclui-se que a maioria dos participantes encontra-se despreparada para o reconhecimento e notificação dessas ocorrências. Sugere-se o aprimoramento das estratégias, de modo a capacitar os profissionais para realizar um atendimento adequado às vítimas de violência intrafamiliar.
\end{abstract}

Descritores: Violência; Saúde Pública; Epidemiologia; Políticas Públicas.

\begin{abstract}
Violence has emerged as an outstanding demand to health sectors. The objective was to verify if the professionals of the Family Health Strategy (FHS) in a city of São Paulo are difficulties concerning the recognition and reporting of incidents involving violence. It is a cross-sectional descriptive study. Of the total of 135 professionals, 57 consented and responded to a self-administered questionnaire with open and closed questions. Data were analyzed quantitatively and qualitatively. $75.4 \%$ of professionals do not know the violence notification form; $60 \%$ do not feel responsible for the reporting of cases. The event of domestic violence often accompanies the use of drugs and / or alcohol as well as the socioeconomic status of the individuals involved. It is concluded that most participants is unprepared to recognize and reporting such occurrences. It is suggested the improvement of strategies in order to train professionals to perform adequate care for victims of domestic violence.
\end{abstract}

Descriptors: Violence; Public Health; Epidemiology; Public Policies.

\section{Resumen}

La violencia se ha convertido en una demanda pendiente con los sectores de salud. El objetivo fue verificar si los profesionales de la Estrategia de Salud de la Familia (ESF) en una ciudad de São Paulo son las dificultades en materia de reconocimiento y notificación de los incidentes relacionados con la violencia. Se trata de un estudio descriptivo de corte transversal. Del total de 135 profesionales, 57 consintieron y respondió a un cuestionario autoadministrado con preguntas abiertas y cerradas. Los datos fueron analizados cuantitativa y cualitativamente. $75,4 \%$ de los profesionales no saben la forma de notificación de la violencia; $60 \%$ no se siente responsable de la notificación de los casos. El caso de la violencia doméstica a menudo acompaña el uso de drogas y / o alcohol, así como la situación socioeconómica de los individuos involucrados. Se concluye que la mayoría de los participantes no está preparado para reconocer y reportar estos hechos. Se sugiere la mejora de las estrategias con el fin de capacitar a los profesionales para llevar a cabo una atención adecuada a las víctimas de la violencia doméstica.

Descriptores: Violencia; Salud Pública; Epidemiología; Políticas Públicas. 


\section{INTRODUÇÃO}

As mortes decorrentes de acidentes e violências são a terceira causa de óbito no Brasil e a primeira na faixa etária do 1 aos $39 \operatorname{anos}^{1}$. Assim a violência é considerada um problema de saúde pública, pelo elevado número de vítimas que atinge e pelos impactos sociais, econômicos e pessoais que provoca ${ }^{2}$.

Neste contexto, a Estratégia de Saúde da Família (ESF) é considerada modelo de reorganização de assistência aos usuários do Sistema Único de Saúde (SUS) e tem sido o lugar privilegiado para trabalhar com esse fenômeno, pois traz em seus objetivos a possibilidade de novas práticas de cuidado a violência, pela aproximação entre usuários e profissionais e a territorialidade, permitindo que os profissionais trabalhem a temática da violência de maneira preventiva e promocional ${ }^{3,4}$.

Infelizmente, muitos trabalhadores da área ainda têm sérias dificuldades para identificar este fenômeno, e na maioria dos casos em que se suspeita de violência, estes não são investigados ${ }^{2,5}$. As causas desta "não percepção" devem-se, provavelmente, a várias circunstâncias como despreparo, desinteresse, falta de tempo para escutar o paciente durante os atendimentos e ao fato de ater-se somente às lesões físicas. Além disso, a maioria das disciplinas dos cursos na área da saúde não contempla em seus currículos a formação nos aspectos relacionados com a violência, e por isso seus egressos não se encontram preparados para oferecer uma atenção que seja efetiva à saúde das vítimas ${ }^{4,6}$.

Um instrumento importante para dimensionar os casos de violência é a notificação, no qual permite estimar a necessidade de investimento em serviços de assistência e o aperfeiçoamento das redes de proteção. Porém, pouco se conhece sobre as iniciativas voltadas para o processo de notificação e qualificação do registro dos casos de violência?

Além de obrigação moral, implícita nos Códigos de Ética das diversas profissões da saúde, a notificação compulsória dos casos de violência dispõe de fortes embasamentos legais ${ }^{8}$.

No entanto, muito ainda há de ser feito para que os objetivos sejam alcançados. Uma coisa é certa, é por meio da notificação que se cria o elo entre a área de saúde e o sistema legal, iniciando-se a formação da rede multiprofissional e interinstitucional da atuação fundamental nesses casos, permitindo também o dimensionamento epidemiológico da violência ${ }^{7}$.

Justifica-se pelo exposto, a realização do estudo, aliada à necessidade de se verificar as principais dificuldades encontradas pelos profissionais de saúde no que se refere ao processo reconhecimento-notificação dos casos de violência.

\section{MATERIAL E MÉTODO}

O projeto foi submetido à apreciação do Comitê de Ética em Pesquisa da Faculdade de Odontologia de Araçatuba - SP - FOA-UNESP, sendo aprovado sob o Processo $n^{\circ}$ 00645/2010.

A realização da pesquisa foi autorizada mediante consentimento do responsável pela Secretaria de Saúde do município. Foram visitadas todas as Unidades Básicas de Saúde (UBS) do município de Araçatuba-SP (n=11) e todos os profissionais da Estratégia de Saúde da Família, foram abordados pessoalmente e convidados a participar da pesquisa.

$\mathrm{O}$ instrumento utilizado consistiu em um questionário autoaplicado, semiestruturado, contendo perguntas abertas e fechadas relativas à notificação de violência, após obtenção do Termo de Consentimento Livre e Esclarecido. O mesmo foi elaborado especialmente para a pesquisa e testado previamente. Depois do teste, o instrumento passou pelas adequações pertinentes. Os dados coletados foram digitados e analisados com auxílio do software estatístico Epi Info versão 3.5.1.

$\mathrm{Na}$ análise qualitativa das questões abertas foi empregada a técnica Análise de Conteúdo ${ }^{9}$, através da categorização das respostas e análise em profundidade dos conteúdos manifestos pelos atores sociais envolvidos. A categorização pode ser definida como uma operação de classificação de elementos constitutivos de conjunto, por diferenciação e, seguidamente, por reagrupamento. As categorias são rubricas ou classes, as quais reúnem um grupo de elementos sob um título genérico, agrupado em razão dos caracteres comuns destes elementos.

A Análise de Conteúdo, como técnica de tratamento de dados, possui a mesma lógica das metodologias quantitativas, uma vez que busca a interpretação cifrada do material de caráter qualitativo $^{10}$. Historicamente a Análise de Conteúdo clássica tem oscilado entre o rigor da suposta objetividade dos números e a fecundidade da subjetividade, dessa forma, em sua origem é vítima da pretensa objetividade que os números e as medidas oferecem $^{10}$.

\section{RESULTADOS}

Do total de 135 profissionais, 57 consentiram em participar da pesquisa. Importante destacar que os responsáveis por duas Unidades Básicas de Saúde não permitiram as entrevistas, mesmo com autorização expressa do Secretário de Saúde do município, o que inviabilizou a coleta de dados nesses locais.

A maioria $(77,2 \%)$ dos entrevistados é composta por agentes comunitários de saúde. Quanto 
ao gênero, 89\% são mulheres e 11\% homens. Quanto ao tempo de serviço na Unidade em que trabalham a maioria (54\%) possui entre $1-5$ anos, $12 \%$ menos de 1 ano, $16 \%$ entre 6-10 anos e $18 \%$ não responderam.

Quando questionados se a violência intrafamiliar é um problema comum na Unidade em que trabalham 38,6\% afirmaram que sim e $61,4 \%$ disseram não ser um problema. Quanto a suspeita de casos de violência intrafamiliar no exercício profissional, 45,6\% dos entrevistados afirmaram já ter suspeitado de algum caso e 54,4\% negou qualquer suspeita.

Quando questionados sobre as causas deste tipo de violência, a maior parte dos entrevistados atribuiu o fato a problemas familiares, como alcoolismo e drogas.

Alcoolismo, drogas, desemprego, família desestruturada, insuficiência monetária, abandono de incapaz, traição.

Falta de diálogo.

Falta de punição para o agressor

Os participantes foram indagados sobre a existência de normas na instituição, relativas a notificação dos casos de violência na instituição em que trabalham. A maioria (58\%) não soube informar, $30 \%$ afirmaram que as normas existem e outros $12 \%$ relataram a inexistência das mesmas.

Sobre o atendimento de casos de violência intrafamiliar, $77 \%$ afirmaram que nunca atenderam casos de violência e $23 \%$ que já atenderam. Em relação aos que atenderam $62 \%$ afirmam que os casos mais comuns envolvem crianças, idosos e mulheres, $23 \%$ atenderam somente idosos nesta situação e outros $15 \%$ tiveram experiência restrita ao atendimento de crianças. Quanto ao questionamento sobre o funcionamento do sistema de registro, a maioria dos profissionais $(56 \%)$ relatou não saber informar por não atender casos de violência, 22\% alegam passar para os seus superiores e estes aos órgãos competentes, $12 \%$ afirmaram que os casos são registrados diretamente nos órgãos competentes, $10 \%$ relataram outras formas.

Uma parte razoável dos profissionais (40\%), relatou não conhecer a legislação para casos de violência intrafamiliar, enquanto $60 \%$ afirmaram conhecê-la. Dos profissionais que afirmaram conhecer a legislação $38 \%$ obtiveram esse conhecimento no local de trabalho, $15 \%$ receberam durante a graduação e $47 \%$ obtiveram por outros meios, como TV, jornais e revistas (44\%), palestras (20\%), internet (12\%), literatura (12\%), pela legislação do Estatuto do idoso $(6 \%)$ e outros $6 \%$ pela assistente social da Unidade.
A respeito do conhecimento sobre a notificação, $56 \%$ dos profissionais desconhecem o que é a notificação de violências e $44 \%$ afirmaram conhecer. Dos profissionais que afirmam saber o que é a notificação $54,2 \%$ obtiveram a informação no local de trabalho, $20,8 \%$ durante a graduação e $25 \%$ por outro meio, sendo que destes, $34 \%$ afirmaram conhecer por meio de palestras e por informações obtidas na Delegacia de Defesa da Mulher, 16\% durante treinamentos e $50 \%$ não souberam informar. No que se refere à ficha de notificação de violência, $75,4 \%$ dos profissionais não a conhecem e $24,6 \%$ dizem conhecê-la. Dos que afirmaram conhecer a ficha de notificação a maioria (50\%) conheceu no local de trabalho, $36 \%$ durante a graduação e $14 \%$ relataram por outros meios, sendo durante treinamentos e na Delegacia de Defesa da Mulher.

Quando questionados sobre a responsabilidade pela notificação dos casos de violência, $60 \%$ acreditam não ter responsabilidade alguma, e $40 \%$ se consideram responsáveis.

Quando questionados quais foram as intervenções feitas no seu setor a maioria diz não atender casos de violência.

Já tive caso de violência passei para meus superiores e não foi tomada nenhuma providência, simplesmente pediu para eu fingir que não vi nada.

A maioria dos casos é omitida, tanto pelo paciente como pela UBS, com isso, fica difícil a intervenção.

\section{Geralmente não há, a não ser eu mesma ligar para} algum familiar.

\section{DISCUSSÃO}

Desde o final da década de 1980 a violência passou a ser considerada um problema de Saúde Pública no Brasil, em decorrência da magnitude da mortalidade $^{11}$. O reconhecimento e a notificação dos casos de violência pelos profissionais de saúde são facilitados, uma vez que eles estão em contato direto com as vítimas de agressões.

Grande parte dos entrevistados, por ser agente comunitário de saúde (ACS), possui um contato muito próximo com a população, o que ajudaria na identificação dos casos de violência já que são eles quem realiza as visitas e repassam os casos para as Unidades Básicas de Saúde (UBSs). Esses profissionais têm a oportunidade de verificar "in loco" as condições da família e acompanhar possíveis ocorrências de violência que não chegam à Unidade.

Um percentual razoável já suspeitou de casos de violência no exercício profissional. A maioria não sabe informar a existência de normas na instituição 
que formalizem a conduta a ser tomada, nessas situações. O conhecimento das normas é importante para a adequada tomada de decisões.

Em relação as causas de violência, os principais fatores, apontado pelos profissionais foi os problemas familiares, no qual envolve o uso de drogas e/ou álcool e também a condição socioeconômica ${ }^{12-14}$.

A maioria afirma que nunca atendeu casos de violência, o que pode ser justificado pelo pouco tempo de serviço (1-5 anos) na Estratégia de Saúde da Família (ESF) e pelo fato de que a maioria dos profissionais contratados é recém-formada. O mesmo motivo pode explicar o fato de não saber informar, como funciona o sistema de registro.

A falta de conhecimento da legislação pelos profissionais pode contribuir para a omissão e, consequentemente, para a ineficácia do instrumento $\operatorname{legal}^{8}$, o que foi observado no estudo já que $40 \%$ relataram não conhecer a legislação referente a violência intrafamiliar.

A notificação insere-se como uma das estratégias primordiais do Ministério da Saúde no âmbito das ações contra a violência e como estratégia para articulação de políticas de saúde ${ }^{15}$. Dessa forma, o profissional de saúde tem o dever de comunicar casos de violência atendidos por $\mathrm{ele}^{8}$, sendo inclusive, obrigatoriamente comunicados ao Conselho Tutelar da respectiva localidade, sem prejuízo de outras medidas legais cabíveis ${ }^{16}$. A falta dessa notificação pode gerar negligência dos serviços de saúde e consequentemente danos ao paciente.

Os profissionais sabem da importância da notificação, como prática estabelecida em sua dinâmica de atendimento, porém, percebe-se que ao serem questionados sobre as fichas, poucos $(24,6 \%)$ sabem da sua existência e quase nenhum realiza seu preenchimento $^{14}$. A disponibilização nos serviços, das fichas de notificação compulsória de violência, é muito importante porque será através delas que os profissionais de saúde passarão a conhecer a situação vivida pelas vítimas, possibilitando assim, o desenvolvimento de ações preventivas e assistências adequadas aos vitimizados.

Estudos também revelam que os profissionais precisam de capacitação específica para a abordagem da violência intrafamiliar ${ }^{5,11,16,17}$. Nesse sentido, as universidades devem participar mais ativamente na formação de profissionais, capacitando-os para o gerenciamento de situações de emergência e atendimento às vítimas de acidentes e de violências, bem como para o desenvolvimento de medidas preventivas $^{12,18}$. Compete também a estas instituições, a realização de estudos a fim de se adotar medidas necessárias à atenção, à prevenção e à promoção de saúde.
Os profissionais sentem receio de se envolver com os casos de violência e acabam se omitindo e também surgi outra questão que é a não resolução do problema pelos órgãos competentes e pelo próprio supervisor, o que causa uma desconfiança desses serviços pelos profissionais ${ }^{16}$.

Em relação as intervenções feita no seu setor em casos de violência, os profissionais relataram uma negligência ao assunto, o que gera uma omissão e a não resolução do problema.

A violência é uma questão invisível nos serviços de saúde, não apenas por um não reconhecimento dos casos por parte dos profissionais, mas por uma negação da existência da mesma, sendo esta uma forma de não lidarem com uma questão para a qual não se sentem capacitados ${ }^{19}$.

A responsabilidade não é somente do profissional e sim da Unidade como um todo, de modo a se construir estratégias que contribuam para a redução da violência, a humanização do serviço de saúde, e a inter-relação com outros serviços de apoio. Para tanto, destaca-se a importância da capacitação da Equipe quanto ao tema, para que os profissionais se aprimorem e desenvolvam a habilidade e a sensibilidade para abordar, reconhecer e notificar, de forma adequada, os casos de violência intrafamiliar.

\section{CONCLUSÃO}

Conclui-se que a maioria dos entrevistados encontra-se despreparado para o reconhecimento e notificação dos casos de violência. Sugere-se que sejam aprimoradas as estratégias para o acolhimento adequado dos casos de violência, de modo a capacitar e sensibilizar os profissionais envolvidos no atendimento às vítimas.

\section{APOIO FINANCEIRO}

Fundação de Amparo à Pesquisa do Estado de São Paulo (FAPESP), Processo 09/53538-2.

\section{REFERÊNCIAS}

1. Brasil. Ministério da Saúde. Secretaria de Vigilância em Saúde. Departamento de Análise de Situação de Saúde. Viva: vigilância de violências e acidentes, 2008 e 2009 / Ministério da Saúde, Secretaria de Vigilância em Saúde, Departamento de Análise de Situação de Saúde. - Brasília: Ministério da Saúde, 2010. 138 p.: il. + 1 CDROM - (Série G. Estatística e Informação em Saúde) Seção 1e.

2. Vieira LB, Padoin SMM, Landerdahl MC. A percepção de profissionais da saúde de um hospital sobre a violência contra as mulheres. Rev Gaúcha Enferm. 2009; 30(4):609-16. 
3. Androulaki Z, Rovithis M, Tsirakos D, Merkouris A, Zedianakis Z, Kakavelakis $\mathrm{K}$ et al. The phenomenon of women abuse: attitudes and perceptions of health professionals working in health care centers in the prefecture of Lasithi, Crete, Greece. HSJ - Health Sci J. 2008;2(1):3344.

4. Guzzo PC, Costa MC, Silva EB, Jahn AC. Práticas de saúde aos usuários em situação de violência: da invisibilidade ao (des)cuidado integral. Rev Gaúcha Enferm. 2014;35(2):100-05.

5. Saraiva RJ, Rosas AMTF, Valente GSC, Viana LO. Qualificação do enfermeiro no cuidado a vítimas de violência doméstica infantil. Cienc. Enferm. 2012;18(1):17-27.

6. Gomes NP, Erdmann AL. Violência conjugal na perspectiva de profissionais da "Estratégia Saúde da Família": problema de saúde pública e a necessidade do cuidado à mulher. Rev Latino-Am. Enfermagem. 2014;22(1):1-9.

7. Deslandes S, Mendes CHF, Lima JS, Campos DS. Indicadores das ações municipais para a notificação e o registro de casos de violência intrafamiliar e exploração sexual de crianças e adolescentes. Cad. Saúde Pública, Rio de Janeiro. 2011;27(8):1633-45.

8. Saliba O, Garbin CAS, Garbin AJI, Dossi AP. Responsabilidade do profissional de saúde sobre a notificação de casos de violência doméstica. Rev Saúde Pública. 2007;41(3):472-77.

9. Bardin, L. Análise de Conteúdo. $3^{\mathrm{a}}$ ed. São Paulo (SP): Ed. 70; 2004.

10. Minayo MCS. O desafio do conhecimento. Pesquisa qualitativa em saúde. São Paulo: Hucitec; 2006.

11. Cavalcanti MLT, Souza ER. Percepções de gestores e profissionais de saúde sobre a atenção aos idosos vítimas de violências no município do Rio de Janeiro (RJ, Brasil). Ciênc. saúde colet. 2010; 15(6):2699-2708.

12. Brasil. Portaria MS/GM n. 936, de 16 de maio de 2001: rede nacional de prevenção da violência e promoção da saúde e a implementação de núcleos de prevenção da violência em estados e municípios. Diário Oficial da União, Brasília, 20 mai 2004.

13. Scaranto CA, Biazevic MGH, Michel-Crosato E. Percepção dos Agentes Comunitários de Saúde sobre a Violência Doméstica contra a Mulher. Psicol. Ciênc. Prof. (Impr). 2007;27(4):694-705.

14. Vieira EM, Perdona GCS, Almeida AM, Nakano AMS, Santos MA, Daltoso D. et al. Conhecimento e atitudes dos profissionais de saúde em relação à violência de gênero. Rev Bras Epidemiol. 2009;12(4):566-77.

15. Veloso MMX, Magalhães CMC, Dell'Aglio DD,
Cabral IR, Gomes MM. Notificação da violência como estratégia de vigilância em saúde: perfil de uma metrópole do Brasil. Ciênc. saúde colet. 2013;18(5):1263-72.

16. Andrade EM, Nakamura E, Paula CS, Nascimento R, Bordin IA. A Visão dos Profissionais de Saúde em Relação à Violência Doméstica Contra Crianças e Adolescentes: um estudo qualitativo. Saúde Soc. São Paulo. 2011;20(1):147-55.

17. Rodrigues DT, Nakano MAS. Violência doméstica e abuso de drogas na gestação. Rev Bras Enferm, Brasília. 2007;60(1):77-80.

18. Souza RG, Santos DV. Enfrentando os maus-tratos infantis nas Unidades de Saúde da Família: atuação dos enfermeiros. Physis. 2013;23(3):783-800.

19. Ferrante FG, Santos MA, Vieira EM. Violência contra a mulher: percepção dos médicos das unidades básicas de saúde da cidade de Ribeirão Preto, São Paulo. Interface (Botucatu). 2009;13(31):287-99.

\section{CONFLITO DE INTERESSES}

Os autores declaram não haver conflitos de interesse.

\section{AUTOR PARA CORRESPONDÊNCIA}

Cléa Adas Saliba Garbin

cgarbin@foa.unesp.br 